\title{
Psychological factors in functional dyspepsia-keeping an open mind
}

A recent study provides evidence that various psychological factors and disturbances in gastric sensorimotor function are associated with different symptom-based subgroups in patients with functional dyspepsia.

According to the Rome III classification, functional dyspepsia can be subdivided into three symptom-based subgroups: postprandial distress syndrome (PDS); nausea and/or vomiting; and epigastric pain syndrome (EPS). “There is controversy in the literature regarding the association of symptom-based subgroups and psychopathology, somatization and gastric sensorimotor function (that is, gastric hypersensitivity and delayed gastric emptying)," says Lukas Van Oudenhove. Previous studies have suggested that gastric sensitivity, psychopathology and somatization are associated with EPS symptoms, and delayed gastric emptying is associated with PDS symptoms. However, another study showed that psychopathology was associated with a diagnosis of PDS.

Van Oudenhove and colleagues used data from 259 patients with functional dyspepsia. First, exploratory and confirmatory factor analyses were performed, which confirmed the existence of the Rome III subgroups. Second, structural equation modelling was used to test the statistical significance of the relationships between depression, somatization, gastric discomfort threshold and gastric emptying on the one hand and the empirically derived symptom-based subgroups on the other.

As expected, somatization was found to be associated with all three symptombased subgroups. "Contrary to what has been shown before and to current clinical beliefs, we found gastric hypersensitivity to be associated with PDS symptoms, and delayed gastric emptying to be associated with EPS symptoms," says Van Oudenhove. "This questions the selection of drugs targeting either gastric hypersensitivity or gastric emptying on the basis of symptom pattern only."

Further research is needed to confirm these findings. In particular, recent and ongoing trials investigating the effects of certain drugs in patients with EPS or PDS will be informative.

\section{Isobel Franks}

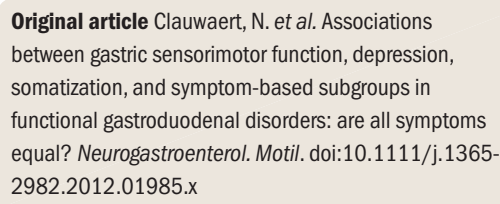

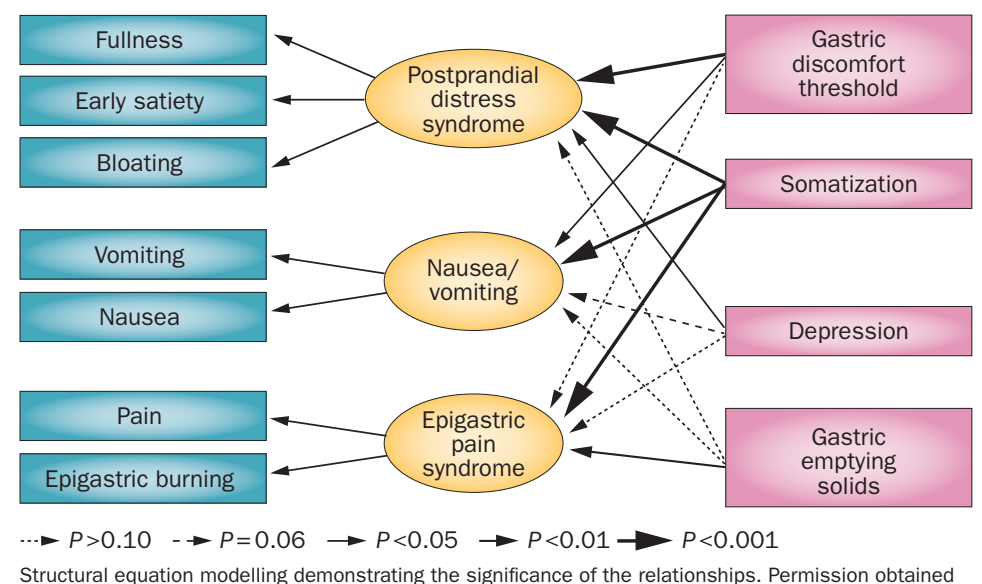

from Wiley @ Clauwaert, N. et al. Neurogastroenterol. Motil. doi:10.1111/j.1365-2982.2012.01985. x 